\title{
Population pharmacokinetic and exploratory exposure-response analysis of the fixed-dose combination of pertuzumab and trastuzumab for subcutaneous injection in patients with HER2-positive early breast cancer in the FeDeriCa study
}

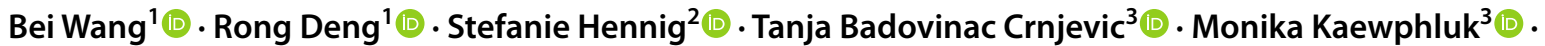

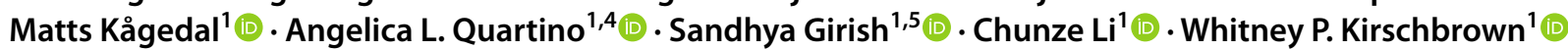

Received: 1 December 2020 / Accepted: 15 May 2021

(C) The Author(s) 2021, corrected publication 2021

\begin{abstract}
Purpose To characterize pertuzumab pharmacokinetics (PK) in FeDeriCa (NCT03493854: fixed-dose combination of pertuzumab and trastuzumab for subcutaneous injection [PH FDC SC] versus intravenous pertuzumab plus trastuzumab); derive individual pertuzumab exposures in the PH FDC SC arm for subsequent pertuzumab exposure-response (ER) analyses; compare observed trastuzumab PK with predicted exposures from a previous SC trastuzumab model; assess whether pertuzumab affects trastuzumab PK; evaluate pertuzumab exposure-efficacy and-safety relationships and support the approved SC dosing regimen.

Methods Population pharmacokinetic modeling and simulations were used to describe the data. Standard goodness-of-fit diagnostics and prediction-corrected visual predictive checks were used for model performance assessment. Covariates were included from previously reported models. ER analysis was conducted using logistic regression.

Results SC pertuzumab PK was described adequately by a two-compartment model with first-order absorption; significant covariates included in the final model were albumin, lean body weight, and Asian region; however, these appeared not to be clinically relevant. Trastuzumab concentrations were described adequately by the previous model; there was no evidence of a pertuzumab effect on trastuzumab PK as part of PH FDC SC and higher model-predicted pertuzumab exposure was not associated with differences in pathologic complete response rate or an increased probability of selected grade $\geq 3$ adverse events of interest.

Conclusion The approved PH FDC SC dose [loading: 1200/600 mg pertuzumab/trastuzumab (15 mL); maintenance: $600 \mathrm{mg}$ pertuzumab/trastuzumab $(10 \mathrm{~mL})$ and $2000 \mathrm{U} / \mathrm{mL}$ recombinant human hyaluronidase every 3 weeks] provides a positive benefit-risk profile with comparable efficacy and safety to intravenous pertuzumab plus trastuzumab.
\end{abstract}

Keywords Exposure-response analysis · Fixed-dose combination $\cdot$ HER2-positive $\cdot$ Pertuzumab, trastuzumab, and hyaluronidase-zzxf $\cdot$ Population pharmacokinetic model $\cdot$ Subcutaneous

The original online version of this article was revised.

Whitney P. Kirschbrown

kirschbrown.whitney@gene.com

1 Genentech, Inc., 1 DNA Way, South San Francisco, CA 94080, USA

2 Certara, Inc., Princeton, NJ, USA

3 F. Hoffmann-La Roche Ltd, Basel, Switzerland

4 Present Address: Clinical Pharmacology and Quantitative Pharmacology, AstraZeneca, Gothenburg, Sweden

5 Present Address: Gilead Sciences, Inc., Foster City, CA, USA

\section{Introduction}

Pertuzumab (PERJETA ${ }^{\circledR}$, F. Hoffmann-La Roche Ltd, Basel, Switzerland) and trastuzumab (Herceptin ${ }^{\circledR}$, F. Hoffmann-La Roche Ltd) have complementary mechanisms of action [1, 2], with in vivo studies showing that they bind to different epitopes on the human epidermal growth factor receptor 2 (HER2) protein. Whereas trastuzumab binds to the extracellular domain of HER2 and inhibits ligand-independent signaling, pertuzumab prevents dimerization of HER2 with other family proteins and, thus, downstream signaling processes associated with tumor growth and progression 
[3-5]. Accordingly, adding intravenous pertuzumab to intravenous trastuzumab (P + H IV) and chemotherapy significantly improves efficacy compared with trastuzumab and chemotherapy in first-line metastatic breast cancer, and in the neoadjuvant and adjuvant early breast cancer settings [6-13].

A fixed-dose combination of pertuzumab and trastuzumab for subcutaneous injection [pertuzumab, trastuzumab, and hyaluronidase-zzxf (PHESGO ${ }^{\mathrm{TM}}$, F. Hoffmann-La Roche Ltd; PH FDC SC)] has been recently approved by several health authorities, including the U.S. Food and Drug Administration (FDA) and the European Medicines Agency. PH FDC SC for the first time combines two monoclonal antibodies in one vial and contains pertuzumab, trastuzumab, and recombinant human hyaluronidase (rHuPH20; an enzyme that temporarily degrades hyaluronan under the skin to aid in the dispersion and absorption of the antibodies) in a ready-to-use formulation. PH FDC SC demonstrates clear time-saving advantages compared with $\mathrm{P}+\mathrm{H} \mathrm{IV}$, as well as other advantages over IV infusion such as less invasiveness, reduced pain, and improved convenience through at-home administration options (via a visiting healthcare professional) [14-16]. The approved SC formulation of trastuzumab has also been shown to have non-inferior serum trough concentrations $\left(\mathrm{C}_{\text {trough }}\right)$ and pathologic complete response (pCR) rates to IV trastuzumab, along with consistent long-term efficacy and a comparable safety profile [17-26]. The pharmacokinetics (PK) of IV pertuzumab, SC pertuzumab, and SC trastuzumab have been studied extensively [27-29] and support their comparable efficacy and safety profiles.

The pertuzumab and trastuzumab doses in the PH FDC SC were supported by population PK (popPK) modeling and simulation data in solid tumors: IV pertuzumab PK was described by a two-compartment linear model with firstorder elimination, with baseline serum albumin and lean body weight (LBW) having statistically significant, but not clinically relevant, effects on IV pertuzumab clearance [27]. A phase Ib study in healthy male volunteers and female patients with breast cancer showed that an SC pertuzumab $600 \mathrm{mg}$ maintenance dose had equivalent exposure $\left[\mathrm{C}_{\text {trough }}\right.$ and area under the curve (AUC)] to IV pertuzumab at $420 \mathrm{mg}$ with no new safety signals [28]. The SC trastuzumab dose within PH FDC SC was based on an analysis from the HannaH study, where a $600 \mathrm{mg}$ fixed dose was confirmed to give the desired exposure [29]. As such, the PH FDC SC is given as a loading dose of $1200 \mathrm{mg}$ pertuzumab and $600 \mathrm{mg}$ trastuzumab in $15 \mathrm{~mL}$, and $600 \mathrm{mg}$ pertuzumab and trastuzumab maintenance doses in $10 \mathrm{~mL}$, along with $2000 \mathrm{U} / \mathrm{mL}$ rHuPH20 every 3 weeks. This dose was assessed clinically in the FeDeriCa study (NCT03493854), which showed that PH FDC SC was non-inferior to P + H IV, based on cycle 7 pertuzumab serum $\mathrm{C}_{\text {trough }}$ concentrations, and that PH FDC SC and P + H IV demonstrated comparable efficacy (total pathologic complete response) and safety profiles [30].

Here, we present three analyses of the FeDeriCa study. First, a popPK analysis aiming to characterize the PK of pertuzumab after P + H IV and PH FDC SC administration and to derive individual pertuzumab exposures in the PH FDC SC arm for subsequent exposure-response (ER) analyses. Second, a comparison of the observed PK of trastuzumab in the PH FDC SC arm with predicted trastuzumab exposures based on a previously established popPK model of SC trastuzumab, to assess whether pertuzumab affects trastuzumab PK in the PH FDC SC arm [29]. Third, a pertuzumab ER analysis in the PH FDC SC arm, which aimed to evaluate the pertuzumab exposure-efficacy and -safety relationships and, thus, confirm the dosing regimen and support the label for this new formulation.

\section{Materials and methods}

FeDeriCa was conducted in conformance with Good Clinical Practice guidelines and the Declaration of Helsinki [30]. The protocol was reviewed and approved by the institutional review board or ethics committee at each study site; all patients provided written informed consent [30].

\section{Study design [30]}

FeDeriCa is a randomized, open-label, international, multicenter, two-arm phase III non-inferiority trial of PH FDC SC compared to $\mathrm{P}+\mathrm{H}$ IV in the neoadjuvant-adjuvant early breast cancer setting. Patients were randomized 1:1 to $\mathrm{P}+\mathrm{H}$ IV or PH FDC SC, with stratification based on hormone receptor status (estrogen receptor- or progesterone receptor-positive, or estrogen receptor- and progesterone receptor-negative), clinical stage (II-IIIA or IIIB-IIIC), and chemotherapy regimen [ 4 cycles of dose-dense doxorubicin plus cyclophosphamide (ddAC) every 2 weeks followed by paclitaxel once weekly for 12 weeks, or 4 cycles of doxorubicin plus cyclophosphamide (AC) every 3 weeks followed by 4 cycles of docetaxel every 3 weeks].

\section{Patients [30]}

Patients had HER2-positive (immunohistochemistry $3+$ or in situ hybridization-positive; centrally confirmed along with hormone receptor status) early breast cancer, were candidates for preoperative neoadjuvant treatment, aged $\geq 18$ years, and had operable, locally advanced, or inflammatory stage II-IIIC disease with a primary tumor $>2 \mathrm{~cm}$ in diameter, or node-positive disease. Patients also had an Eastern Cooperative Oncology Group (ECOG) performance 
status of 0 or 1 and a left ventricular ejection fraction of $\geq 55 \%$ (by echocardiography or multiple-gated acquisition scan). Exclusion criteria included receipt of any systemic therapy for treatment or prevention of breast cancer, or radiation treatment for cancer, serious cardiac conditions, impaired liver function, or inadequate renal or bone marrow function.

\section{Procedures [30]}

Investigators chose one of two chemotherapy regimens before randomization: 4 cycles of ddAC comprising $60 \mathrm{mg} /$ $\mathrm{m}^{2}$ doxorubicin plus $600 \mathrm{mg} / \mathrm{m}^{2}$ cyclophosphamide every 2 weeks (with local guideline-specified granulocyte colony-stimulating factor if needed, per local guidelines) followed by $80 \mathrm{mg} / \mathrm{m}^{2}$ paclitaxel once a week for 12 weeks, or 4 cycles of $\mathrm{AC}$ comprising $60 \mathrm{mg} / \mathrm{m}^{2}$ doxorubicin plus $600 \mathrm{mg} / \mathrm{m}^{2}$ cyclophosphamide every 3 weeks followed by 4 cycles of $75-100 \mathrm{mg} / \mathrm{m}^{2}$ docetaxel every 3 weeks. Following anthracycline-based chemotherapy, and concomitant with the taxane chemotherapy, patients received four cycles of HER2-targeted therapy (given before the taxane on the same day). P IV was given as an $840 \mathrm{mg}$ loading dose followed by $420 \mathrm{mg}$ maintenance doses every 3 weeks; $\mathrm{H}$ IV, as an $8 \mathrm{mg} / \mathrm{kg}$ loading dose followed by $6 \mathrm{mg} / \mathrm{kg}$ maintenance doses every 3 weeks (the order being according to investigator preference). PH FDC SC was given as a $1200 \mathrm{mg}$ $\mathrm{P} / 600 \mathrm{mg} \mathrm{H}$ loading dose in $15 \mathrm{~mL}$ followed by $600 \mathrm{mg}$ $\mathrm{P} / 600 \mathrm{mg} \mathrm{H}$ maintenance doses in $10 \mathrm{~mL}$ every 3 weeks. After completion of this neoadjuvant component of the study, patients received surgery, followed by 14 three-weekly HER2-targeted therapy cycles as randomized to complete a maximum total of 18 cycles. Treatment was discontinued for instances of investigator-assessed radiographic or clinical disease progression or recurrence or unmanageable toxicity. Dose modifications of PH FDC SC, P IV, or H IV were not allowed. Patients were withdrawn from all study treatment if the HER2-targeted component was withheld for more than 2 cycles (i.e., more than 9 weeks between doses) or if HER2-targeted therapy needed to be permanently discontinued for treatment-related toxicity.

This analysis is based on the neoadjuvant period only. During this period, blood samples for PK assessments were collected pre- and post-dose on day 1 in the $\mathrm{P}+\mathrm{H}$ IV arm in cycles $5,6,7,8$, and on days $2,4,8$, and 15 of cycle 7 . In the PH FDC SC arm, samples were collected pre-dose on day 1 of cycles $5,6,7$, and 8 , on days 2 and 15 of cycle 5 , and on days $2,4,8$, and 15 of cycle 7. Actual collection times were recorded. A validated duplex hybrid immunoaffinity capture liquid chromatography tandem mass spectrometry assay was used to simultaneously measure the concentration of both pertuzumab and trastuzumab in serum samples. The concentration of the lower limit of quantification (LLQ) for both analytes was $100 \mathrm{ng} / \mathrm{mL}$.

\section{Pertuzumab population pharmacokinetic analysis}

PK analyses included both SC and IV data and patients. Patients were included in the popPK analysis if they had at least one PH FDC SC or P + H IV administration and a corresponding PK sample collection after dosing with a concentration above the LLQ. Samples were included from cycle 5 through to day 1 of cycle 8 . If the time of either drug administration or sample collection was missing, the record was excluded from analysis. Patients who had at least one observed post-dose pertuzumab concentration and are included as part of the final popPK model were included in the ER analysis (patients in the PH FDC SC arm only). Any observations below the LLQ, of which there were 16 $(0.31 \%)$, were omitted across datasets.

A two-compartment model with first-order absorption for SC administration and first-order elimination from the central compartment was utilized as a starting point for the modeling using NONMEM version 7.4.3 (ICON Development Solutions, Ellicott City, Maryland, USA) with the first-order conditional estimation with interaction method. Evaluated covariates included those from previous pertuzumab IV models (LBW, albumin), standard demographic variables (age, sex, race), standard baseline laboratory variables (aspartate aminotransferase, alanine aminotransferase, total protein, total bilirubin, alkaline phosphatase, creatinine, creatinine clearance), stratification factors in the study design [hormone receptor status (estrogen receptor- or progesterone receptor-positive, or estrogen receptor- and progesterone receptor-negative), clinical stage (II-IIIA or IIIB-IIIC), and chemotherapy regimen ( 4 cycles of ddAC every 2 weeks followed by paclitaxel once weekly for 12 weeks, or 4 cycles of AC every 3 weeks followed by 4 cycles of docetaxel every 3 weeks)], standard disease-related variables (ECOG), or covariates of specific interest (Asian region). Covariates were selected using a forward addition and backward elimination stepwise procedure (significance levels set to $p<0.01$ and $p<0.001$, respectively). Potential outliers were excluded based on conditional-weighted residuals ( $\mid$ CWRES $\mid>5$ ) after the initial base model assessment. Models were evaluated using standard goodness-of-fit diagnostics and prediction-corrected visual predictive checks (pcVPC). Model applications included a display of the magnitude of covariate effects using forest plots, and exposure-comparisons using post hoc exposure estimates stratified by covariates of interest. 


\section{Trastuzumab population pharmacokinetic simulations for assessment of drug-drug interactions}

The H PK dataset included patients from the FeDeriCa study who had at least one administration of PH FDC SC and one post-dose trastuzumab PK sample above the LLQ. Samples were included from cycle 5 through to day 1 of cycle 8 . The HannaH popPK model was a two-compartment model with parallel linear and nonlinear elimination, and first-order absorption for SC administration [29]. This model was used to simulate trastuzumab exposures based on the FeDeriCa study design and patient characteristics (dosing regimens, baseline covariates, and sample times). Trastuzumab concentration predictions from the HannaH PK model and the observed trastuzumab concentrations from FeDeriCa were then compared visually via a pcVPC and numerically using a numerical predictive check (NPC). This was done using NONMEM version 7.4.3 and Perl Speaks NONMEM version 4.8.1 (Uppsala University, Sweden).

\section{Exposure-response and exposure-safety analyses}

The datasets included all patients who received PH FDC SC and had one post-dose pertuzumab concentration above the LLQ. The post hoc PK parameter estimates from these patients were used to generate popPK model-predicted exposures. Exposure metrics included model-predicted cycle 7 $\mathrm{C}_{\text {trough }}$ (pre-dose cycle 8) for efficacy analyses, cycle 7 maximum concentration $\left(\mathrm{C}_{\max }\right)$ for safety analyses, and cycle 7 AUC for both. Cycle 7 represents three doses of pertuzumab (1200 mg loading dose at cycle 5 and $600 \mathrm{mg}$ maintenance dose every 3 weeks thereafter), and based on PK simulation, the majority of patients reached $\mathrm{C}_{\text {trough }}$ steady state at cycle 7. Exploratory analysis included exposure boxplots versus event status and event rates versus mean exposure stratified by exposure quartiles. Modeling was conducted using logistic regression ( $\mathrm{R}$ version 3.4.3). If an ER relationship was observed, covariate testing was performed using a forward-backward stepwise search method (significance levels of $p<0.05$ and $p<0.01$, respectively). Covariates included standard demographic variables (age, sex, race), ECOG performance status, and study stratification variables. Response rates were projected for populations and in different exposure quartiles relative to the overall population, for endpoints with a significant exposure-efficacy relationship. Safety events used in this analysis included grade $\geq 3$ adverse events (AEs), serious AEs, grade $\geq 3$ diarrhea, grade $\geq 3$ neutropenia, cardiac toxicity, injection-related reactions, and hypersensitivity; all of which occurred between cycle 5 and cycle $7 \mathrm{C}_{\text {trough }}$ measurement.

\section{Results}

\section{Patients and samples}

Five hundred patients were randomized [30], and 5656 pertuzumab serum PK samples were collected from 489 patients. A summary of baseline demographics and clinical characteristics is shown in Online Resource 1. Nine patients were not sampled, one had no post-dose sample above the LLQ, and one did not provide PK samples in cycles 5 to 8 . Of the 489 patients with pertuzumab PK samples, 246 (50.3\%) were randomized to the P+H IV arm and $243(49.7 \%)$ to the PH FDC SC arm. After excluding pre-dose pertuzumab serum PK samples prior to the first dose in cycle $5(n=460)$, a further $16(0.31 \%)$ samples below the limit of quantification (seven in the PH FDC SC arm) were identified and removed. This resulted in a total of 5180 evaluable pertuzumab serum PK samples for the analysis, of which 2093 observations were after SC injection and 3087 after IV infusion.

\section{Pertuzumab population pharmacokinetic analysis}

Overall, 40/5180 (0.8\%) PK samples were excluded from the analysis based on conditional-weighted residuals (|CWRES|> 5) after the initial base model assessment (an initial 2-compartment model with linear elimination from the central compartment, first-order absorption of the SC formulation, and a proportional residual error model); among them, there were $7(0.14 \%)$ outliers after SC administration and $33(0.64 \%)$ outliers after IV administration. The final model building dataset contained 489 patients and 5140 pertuzumab serum PK samples. In the forward stepwise covariate modeling step, in addition to LBW on clearance (CL), central volume $\left(V_{c}\right)$, and peripheral volume $\left(V_{p}\right)$ and albumin on $\mathrm{CL}$, Asian (region) was added as significant covariate $(p<0.01)$ on CL. No covariate was excluded through the backward step. Final model parameters are summarized in Table 1. Covariate relationships and parameter values are described by the equations below; the final bioavailability $(F)$ was 0.712 :

$$
\begin{aligned}
\mathrm{CL}(\mathrm{L} / \text { days })= & 0.163 \times(\mathrm{ALB} / 43.25)^{-0.629} \times(\mathrm{LBW} / 45.09)^{1.252} \\
& \times(1.123 \text { if in Asian region }) \times e^{\eta C L}, \\
V_{c}(\mathrm{~L})=2.77 & \times(\mathrm{LBW} / 45.09)^{0.839} \times e^{\eta V 2}, \\
Q(\mathrm{~L} / \text { days })= & 0.616,
\end{aligned}
$$$$
V_{p}(\mathrm{~L})=2.49 \times(\mathrm{LBW} / 45.09)^{0.716} \times e^{\eta V 3},
$$$$
F=0.712 \times e^{\eta F} .
$$ 
Table 1 Final population pharmacokinetic model parameter estimates

\begin{tabular}{|c|c|c|c|c|c|c|}
\hline Parameter & Parameter description & Estimate $(95 \% \mathrm{CI})$ & $\begin{array}{l}\text { RSE for } \\
\text { estimate, \% }\end{array}$ & IIV, CV\% (95\% CI) & RSE for IIV, $\%$ & Shrinkage, $\%$ \\
\hline$\theta_{1}$ & $\mathrm{CL}, \mathrm{L} / \mathrm{d}$ & $0.163(0.144,0.182)$ & 5.81 & $23.5(4.89,32.8)$ & 48.8 & 14.4 \\
\hline$\theta_{2}$ & $V_{c}, \mathrm{~L}$ & $2.77(2.60,2.94)$ & 3.15 & $34.8(32.1,37.3)$ & 7.52 & 11.2 \\
\hline$\theta_{3}$ & $Q, \mathrm{~L} / \mathrm{d}$ & $0.616(0.553,0.679)$ & 5.23 & - & - & - \\
\hline$\theta_{4}$ & $V_{p}, \mathrm{~L}$ & $2.49(2.11,2.87)$ & 7.69 & $25.6(23.5,27.5)$ & 7.93 & 49.7 \\
\hline$\theta_{5}$ & $k_{\mathrm{a}}, \mathrm{d}$ & $0.348(0.295,0.401)$ & 7.72 & - & - & - \\
\hline$\theta_{6}$ & $F$ & $0.712(0.489,0.935)$ & 16.0 & $17.8(17.6,18.0)$ & 1.10 & 50.2 \\
\hline \multicolumn{7}{|c|}{ Residual error } \\
\hline$\theta_{7}$ & Proportional residual error (SC) & $0.155(0.142,0.168)$ & 4.32 & - & - & 10.1 \\
\hline$\theta_{8}$ & Proportional residual error (IV) & $0.175(0.160,0.190)$ & 4.41 & - & - & 10.1 \\
\hline \multicolumn{7}{|l|}{ Covariates } \\
\hline$\theta_{9}$ & Albumin on CL & $-0.629(-0.221,-1.04)$ & 33.1 & - & - & - \\
\hline$\theta_{10}$ & LBW on $\mathrm{CL}$ & $1.25(0.922,1.58)$ & 13.4 & - & - & - \\
\hline$\theta_{11}$ & LBW on $V_{c}$ & $0.839(0.545,1.13)$ & 17.9 & - & - & - \\
\hline$\theta_{12}$ & LBW on $V_{p}$ & $0.716(0.251,1.18)$ & 33.1 & - & - & - \\
\hline$\theta_{13}$ & Change of CL in the Asian region & $0.123(0.049,0.197)$ & 31.0 & - & - & - \\
\hline Half-life, d & & 24.3 & - & 28.2 & - & - \\
\hline
\end{tabular}

The half-life estimate was calculated from typical parameter values. The half-life IIV was calculated from post hoc estimates

$C L$ is clearance, $C V$ is coefficient of variation, $d$ is days, $F$ is bioavailability, $I V$ is inter-individual variability, $I V$ is intravenous, $L B W$ is lean body weight, $k$ a is apparent first-order absorption, $Q$ is inter-compartmental clearance, $R S E$ is relative squared error, $S C$ is subcutaneous, $V_{c}$ is central volume, $V_{p}$ is peripheral volume

Parameters were well estimated with all relative standard errors $<35 \%$ for fixed-effects parameters (Table 1 ).

CL decreased in patients with higher albumin concentrations and increased in patients with greater LBW. Both $V_{c}$ and $V_{p}$ increased in patients with greater LBW. CL was approximately $10 \%$ greater in patients in the Asian region. All exposure ratios fell within the $0.8-1.25$ boundary relative to a typical patient, except for a patient at the 5th percentile LBW of $38 \mathrm{~kg}$ relative to a typical patient with LBW $45.1 \mathrm{~kg}$ (ratio of 1.26) (Fig. 1).

As expected, heavier patients had lower exposures as described by the LBW effect on CL (Fig. 2). Although $\mathrm{CL}$ was greater in the Asian region, patients in that region had similar trough levels to patients in the non-Asian region, because their LBW was lower. Median predicted cycle $7 \mathrm{C}_{\text {trough }}$ concentrations for patients $\leq 65$ years was $82.7 \mu \mathrm{g} / \mathrm{mL}$ (range, $9.0-208.6 \mu \mathrm{g} / \mathrm{mL}$ ) versus $80.8 \mu \mathrm{g} / \mathrm{mL}$ $(32.7-156.9 \mu \mathrm{g} / \mathrm{mL}$ ) for older patients (43 out of 489 were $>65$ years). All (243/243) patients had trough levels above $20 \mu \mathrm{g} / \mathrm{mL}$ in the PH FDC SC arm and $99.6 \%$ (245/246) in the P+ H IV arm.

Goodness-of-fit showed good agreement between predicted and observed concentrations, with no apparent bias in residuals over time and across predicted concentration values (Online Resource 2). The pcVPC for the model is shown in Fig. 3. The central tendency of the model and the 95th percentile ranges were comparable with the data for both the P+H IV and PH FDC SC cohorts.

\section{Pertuzumab-trastuzumab drug-drug interactions assessment}

A total of 243 patients receiving PH FDC SC had 2092 evaluable trastuzumab samples. Overall, the central tendency and range of predicted trastuzumab concentrations by the HannaH model were comparable to those observed from the FeDeriCa data (Online Resource 3); the HannaH study collected trastuzumab samples at earlier time points compared with FeDeriCa and HannaH data appeared to have greater variability at the 95 th percentile prediction range within the first 15 days after the dose.

NPC results based on 1000 simulations showed close agreement between the HannaH model and the FeDeriCa data: $1.9 \%$ of observations were above the 95 th percentile, $48.8 \%$ above the 50th percentile, and $4.5 \%$ below the 5 th percentile. 
a

Covariate

Lean body weight $(\mathrm{kg})$

5th 38

$\begin{array}{cc}\text { 5th } & 38 \\ \text { 25th } & 42 \\ \text { 75th } & 49 \\ \text { 95th } & 53\end{array}$

Baseline albumin $(\mathrm{g} / \mathrm{L})$

Region

\section{Percentile Value}

Ratio

0.91

0.81

0.93

0.97

1.03

1.06

0.89

b

\begin{tabular}{|c|c|c|c|c|c|}
\hline Covariate & Percentile & Value & & & Ratio \\
\hline \multicolumn{6}{|c|}{ Lean body weight $(\mathrm{kg})$} \\
\hline & 5 th & 38 & & $\bullet$ & 1.2 \\
\hline & 25th & 42 & & $\bullet$ & 1.08 \\
\hline & 75th & 49 & $\bullet$ & & 0.92 \\
\hline & 95th & 53 & $\bullet$ & & 0.84 \\
\hline \multicolumn{6}{|c|}{ Baseline albumin (g/L) } \\
\hline & 5th & 38 & $\bullet$ & & 0.95 \\
\hline & 25th & 41 & $\bullet$ & & 0.98 \\
\hline & 75th & 46 & & $\bullet$ & 1.02 \\
\hline & 95th & 48 & & $\bullet$ & 1.04 \\
\hline \multirow[t]{2}{*}{ Region } & & Asian & $\bullet$ & & 0.93 \\
\hline & & 0.6 & $\begin{array}{l}0.8 \\
\text { Ratio relative to }\end{array}$ & a typical subject & 1.4 \\
\hline
\end{tabular}

c

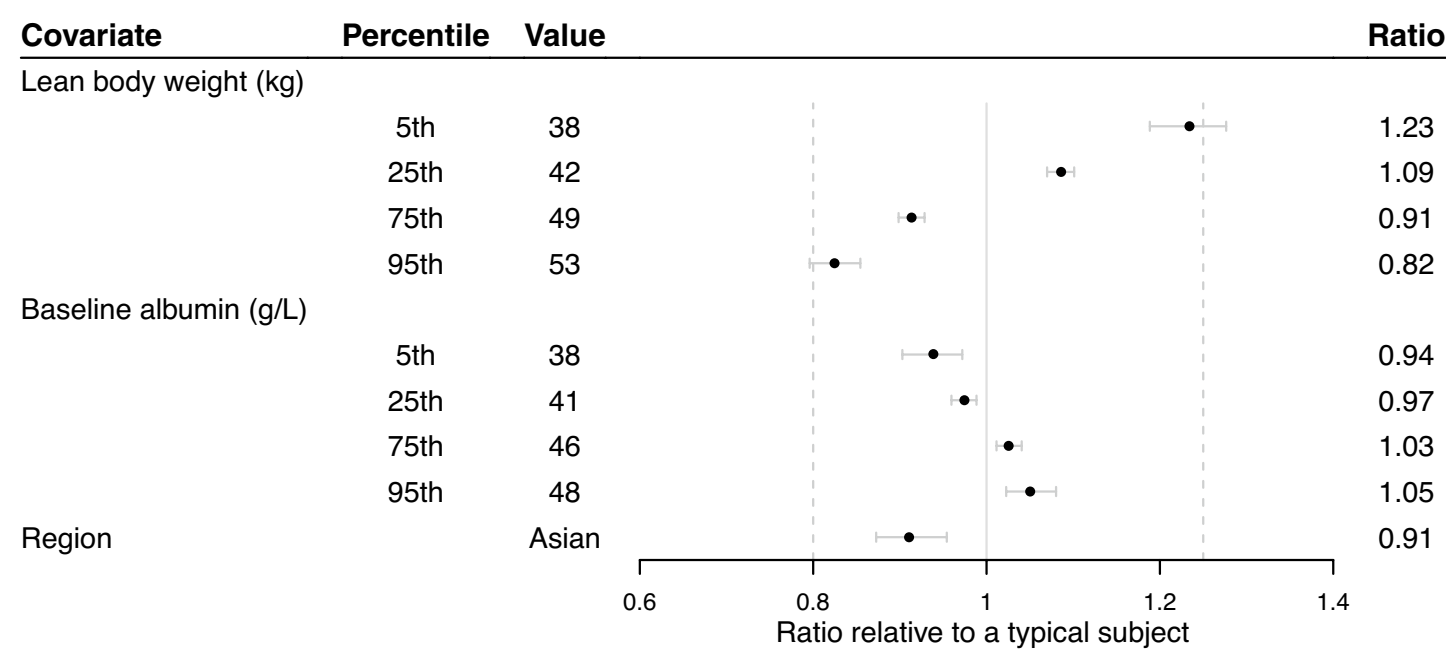


4 Fig. 1 Effects of covariates on pertuzumab exposure metrics $\left[\mathrm{C}_{\text {trough }}\right.$ (A), $\mathrm{C}_{\max }(\mathbf{B})$, and AUC (C)] at cycle 7. Points represent the estimated exposure ratio for the patient with indicated covariate value relative to a typical patient with lean body weight $45.1 \mathrm{~kg}$, albu$\min 43.3 \mathrm{~g} / \mathrm{L}$, who is non-Asian, receiving a dose of pertuzumab at $1200 \mathrm{mg}$ in cycle 5 and $600 \mathrm{mg}$ in cycles 6 and 7 as part of their PH FDC SC administration. The horizontal bars represent $90 \%$ confidence intervals. The vertical dashed lines at 0.8 and 1.25 indicate standard bioequivalence ratio bounds. The vertical line at one indicates a null effect. $A U C$ is area under the curve for a dosing interval $(\mathrm{tau}=21) . C_{\max }$ is maximum concentration. $C_{\text {trough }}$ is trough concentration. Created using R version 3.6 .0 (2019-04-26) in RStudio version 1.2.1335

\section{Pertuzumab exposure-efficacy of PH FDC SC}

All 243 patients in the PH FDC SC arm with model-predicted pertuzumab cycle $7 \mathrm{C}_{\text {trough }}$ were included in the analysis. Patients who achieved total pathologic complete response (tpCR; eradication of invasive disease in the breast and axilla, according to local pathologist assessment [ypT0/ is, ypN0]) had a slightly higher median model-predicted pertuzumab cycle $7 \mathrm{C}_{\text {trough }}(92 \mu \mathrm{g} / \mathrm{mL})$ compared with those who did not $(86 \mu \mathrm{g} / \mathrm{mL})$ (Fig. 4A). Regardless of this difference in exposure, overall tpCR rates in the ER population were comparable: $60.9 \%$ in the PH FDC SC arm and $61.0 \%$ in the P + H IV arm. Median model-predicted AUC was similar in patients who achieved tpCR $(2607 \mu \mathrm{g} . \mathrm{d} / \mathrm{mL}$ versus $2532 \mu \mathrm{g} . \mathrm{d} / \mathrm{mL}$ ) (Fig. 4B).

There was no clear trend in tpCR across pertuzumab exposure quartiles (Table 2). Logistical regression for tpCR in the PH FDC SC arm indicated a slightly positive ER trend in the univariate case $(p=0.048)$ and after adjusting for baseline hormone receptor status ( $p=0.069)$ (Fig. 4C). Differences in tpCR from the overall mean tpCR in the IV arm were small and ranged from -7.6 to $7.4 \%$ in the second and third quartiles, respectively. After adjusting for baseline hormone receptor status, there was no statistically significant relationship between model-predicted pertuzumab exposures and tpCR rate $(p=0.069)$.

\section{Exposure-safety of PH FDC SC}

There was a slightly higher rate of serious AEs in patients with lower exposures (1st and 2nd quartiles of model-predicted pertuzumab cycle $7 \mathrm{C}_{\max }$ ) compared with patients with higher exposures (3rd and 4th quartiles) (Table 2). Higher $\mathrm{C}_{\max }$ quartiles were not associated with higher $\mathrm{AE}$ rates; accordingly, no trends with exposure were observed for grade $\geq 3$ AEs, serious AEs, grade $\geq 3$ diarrhea, grade $\geq 3$ neutropenia, cardiac events, injection-related reactions of any grade within $24 \mathrm{~h}$ related to PH FDC SC, or any hypersensitivity/anaphylaxis related to PH FDC SC. Similar results were observed for cycle 7 AUC (data not shown).

\section{Discussion}

Pertuzumab popPK following PH FDC SC or P + H IV administration in FeDeriCa was described adequately by a two-compartment model with first-order absorption. Statistically significant covariates on pertuzumab exposure (cycle $7 \mathrm{C}_{\text {trough }}$ ) included LBW, race, and albumin; however, their effects were relatively small compared with the overall interindividual variability of the population and the effects were not considered clinically relevant. Thus, no dose adjustments of PH FDC SC based on these covariates are warranted. As described above, cycle 7 was selected for pertuzumab exposure metrics, as this was when the majority of patients reached steady state. When investigating lean LBW, heavier patients had a lower exposure, and lighter patients a higher exposure, due to fixed dosing, as expected. The interquartile range of exposures, as well as the whiskers of the plots, were all greatly overlapping across LBW quartiles. There was no apparent relationship suggestive of an increase in severe or serious AE rates with increasing exposure or decreasing body weight for H SC [29]. Overall, similar to the experience with $\mathrm{P}+\mathrm{H}$ IV/SC, there did not appear to be an impact of LBW on safety of PH FDC SC; however, the low numbers of patients/events in the FeDeriCa subgroups preclude definitive conclusions. Additionally, even in heavier patients (LBW > $48.8 \mathrm{~kg}$ ), all patients following PH FDC SC administration achieved the target efficacious steady-state trough concentration of $20 \mu \mathrm{g} / \mathrm{mL}$ at pre-dose cycle 8 .

Furthermore, similar to the previous $\mathrm{P}+\mathrm{H}$ IV analysis [31, 32], PH FDC SC administration did not show a clinically meaningful ER relationship between tpCR and $\mathrm{AE}$ rates, and was found to demonstrate a manageable safety profile and a comparable efficacy to $\mathrm{P}+\mathrm{H}$ IV.

The FeDeriCa $\mathrm{P}$ population PK model is a two-compartment model with first-order SC absorption with estimated bioavailability of $71 \%$. The bioavailability estimated here was consistent with the findings from the phase Ib SC pertuzumab dose-finding study [28]. The high shrinkage for F that was observed was expected, as only half of the population had IV data and none of the patients received both the IV and SC formulations.

The model demonstrates a smaller clearance $(0.163 \mathrm{~L} / \mathrm{d}$ for current model; $0.235 \mathrm{~L} / \mathrm{d}$ for previous model) and similar volume estimates than previous estimates for $\mathrm{P}+\mathrm{H} \mathrm{IV}$, based on a dataset with a slightly smaller median LBW and larger median albumin [27]. The estimated pertuzumab clearance 
Fig. 2 Model-predicted cycle $7 \mathrm{C}_{\text {trough }}$ by lean body weight quartile $(\mathbf{A})$ and Asian region (B). Solid bold lines represent medians. Shaded boxes represent the interquartile ranges. Whiskers represent 1.5 times the interquartile range. Points are jittered in the $\mathrm{x}$-axis direction for clarity. $\mathrm{C}_{\text {trough }}$ were generated using individual empirical Bayes estimates.

$C_{\text {trough }}$ is trough concentration. Created using $\mathrm{R}$ version 3.6.0 (2019-04-26) in RStudio version 1.2.1335 $\mathbf{a}$

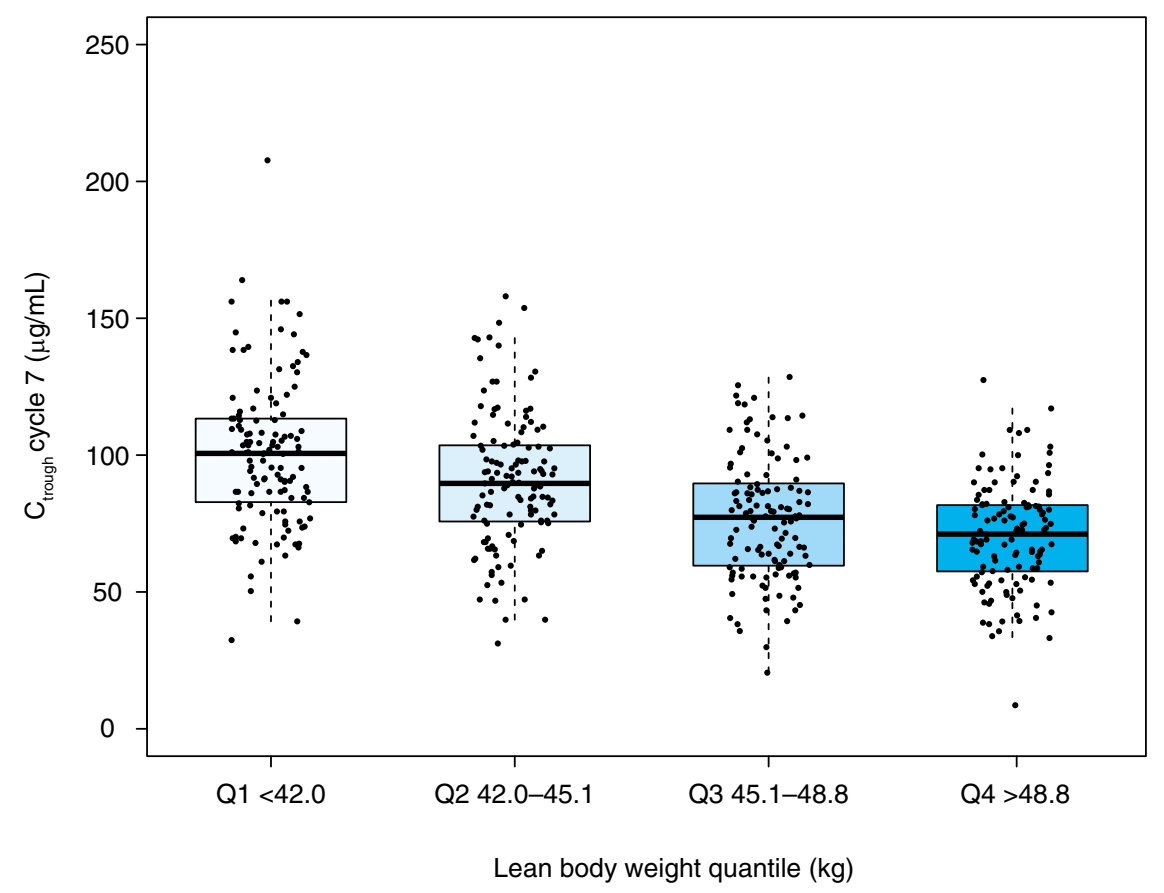

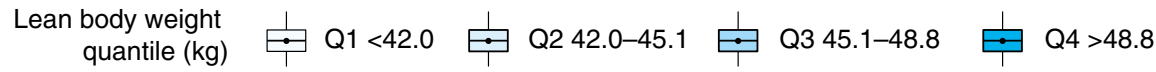

b

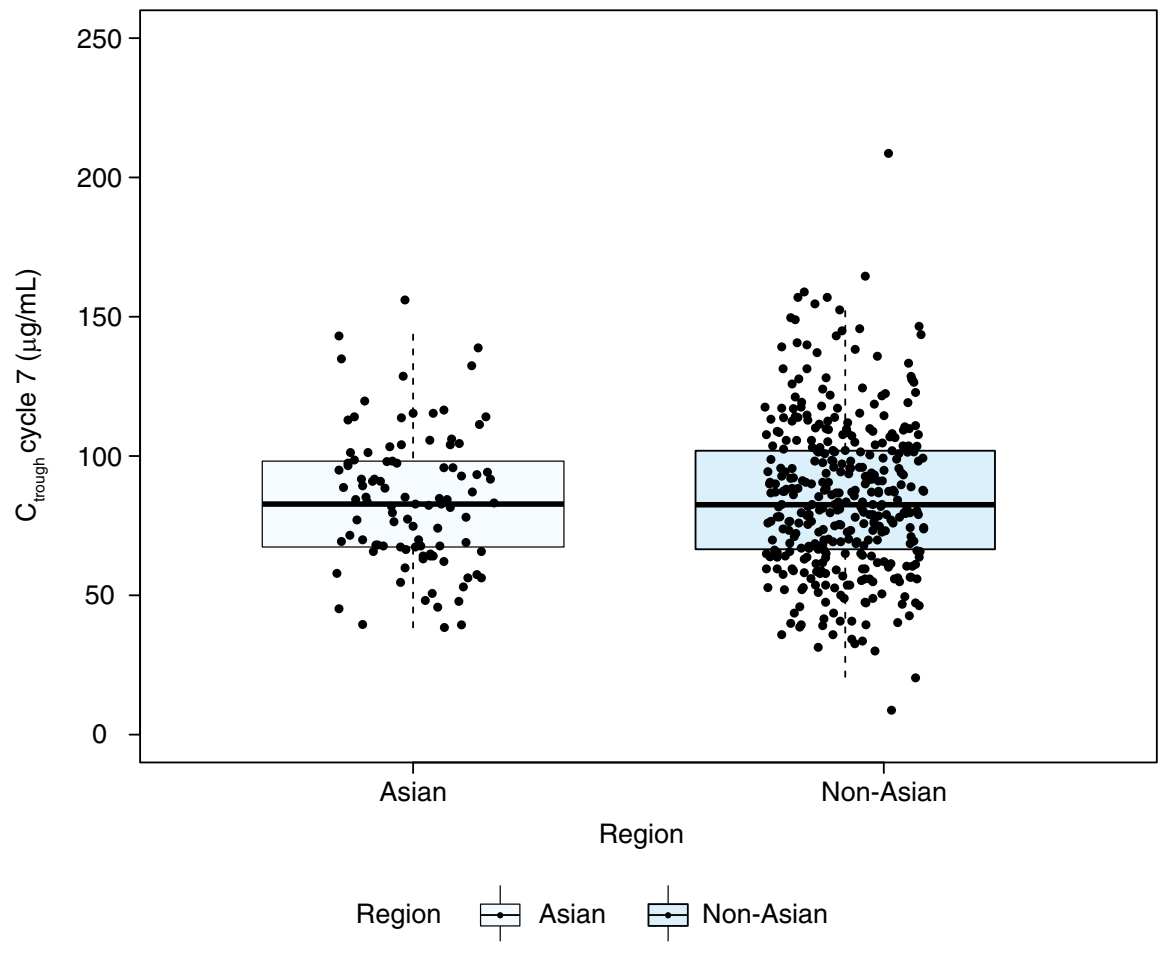


for a typical patient in the FeDeriCa study using the previous P IV model [27] is $0.204 \mathrm{~L} / \mathrm{d}$.

Similar to previous studies of PK of P + H IV administration, baseline serum albumin and LBW were found not to be clinically relevant for pertuzumab exposure for PH FDC SC, indicating that variations in these covariates do not require dose adjustments. In the current study, the Asian region was associated with $12 \%$ increase in CL; in contrast to the 100 Asian patients included here, the previous IV pertuzumab popPK analysis in 2014 had only 22 (all of which were Japanese), and did not appear to impact on CL in a similar way. Nevertheless, in the present study, a similar $\mathrm{CL}$ and exposure in Asian patients compared to the overall population is expected when accounting for the lower LBW of this subgroup.

Trastuzumab concentrations in the PH FDC SC arm were described adequately by the previously developed trastuzumab popPK model from HannaH data [29]. The NPC showed that $\sim 50 \%$ of observed trastuzumab concentrations in the PH FDC SC arm of the FeDeriCa study were above and below the 50th percentile prediction from the HannaH population model [29], suggesting that pertuzumab does not affect trastuzumab PK as part of the PH FDC SC. This was consistent with IV data for the combination [27, 31, 32] and may be due to the fact that HER2 epitope binding between the two antibodies is different with no steric hindrance (meaning that target-mediated drug-drug interactions were unlikely) [1]. It may also be due to the fact that target-mediated drug-drug interactions are saturated at the current dose of PH FDC SC and both monoclonal antibodies are eliminated through largecapacity, non-specific, Fc receptor-mediated immunoglobulin $\mathrm{G}$ clearance mechanisms [33].

Differences in tpCR rate were not associated with differences in median model-predicted pertuzumab cycle 7 $\mathrm{C}_{\text {trough }}$, and the response across all exposure quartiles was similar to that in the $\mathrm{P}+\mathrm{H}$ IV arm. In a similar analysis of the NeoSphere study, which utilized P + H IV, there was no association between the pertuzumab serum concentration and pCR in patients with HER2-positive breast cancer [31]. In the present study, lower rates of tpCR were observed in patients with hormone receptor-positive status, but this trend was not statistically significant. Therefore, the detected numerical difference should be interpreted with caution; this observation may be attributed to: (1) the ten-fold range of $\mathrm{P}$ exposures (21-209 $\mu \mathrm{g} / \mathrm{mL})$ in the PH FDC SC arm; (2) the relatively large sample size $(n=243)$ compared with previous IV-based studies; or (3) may be an artifact caused by a confounding disease status factor that could influence both exposure and response, which have been observed for other biologics for oncology indications (although this is not normally the case in the early breast cancer setting) $[32,34]$.

All patients in the PH FDC SC arm achieved modelpredicted $\mathrm{C}_{\text {trough }}$ exceeding $20 \mu \mathrm{g} / \mathrm{mL}$, which is the targeted effective concentration identified from preclinical mouse xenograft models and early clinical response data [27]. In the present study, higher model-predicted pertuzumab cycle $7 \mathrm{C}_{\max }$ and AUC were not associated with increased probability of grade $\geq 3$ AEs or increased probability of cardiac toxicity, serious AEs, grade $\geq 3$ diarrhea, grade $\geq 3$ neutropenia, injection-related reactions (any grade) within $24 \mathrm{~h}$ related to PH FDC SC, nor was hypersensitivity/anaphylaxis (any grade) related to PH FDC SC.

The ER of SC trastuzumab was well characterized in the HannaH study [29]. In HannaH, fixed $600 \mathrm{mg}$ SC dose of trastuzumab provided the desired exposure, with steady-state $\mathrm{C}_{\text {trough }}(35-123 \mu \mathrm{g} / \mathrm{mL}$ for the 5 th-95th percentiles) above the historical target concentration of $20 \mu \mathrm{g} / \mathrm{mL}$ for efficacy. Fixed dosing was further supported by lack of an ER relationship between PK, tpCR, and grade $\geq 3$ AEs, and no dose adjustments were required for covariates such as albumin or LBW.

Limitations of the current analysis include a limited number of AEs (therefore, results should be interpreted cautiously) and the fact that only one dose level was observed and studied, which limits our ability to describe the exposure-efficacy and exposure-safety relationships. In addition, while shrinkage for CL and $V_{c}$ was low, shrinkage for $F$ and $V_{p}$ were higher, which may have impacted the modelpredicted exposures.

Overall, available popPK and ER analyses for the FeDeriCa study, in conjunction with clinical experience with IV pertuzumab, IV trastuzumab, and SC trastuzumab, indicates that the current dose of PH FDC SC (loading dose: $1200 \mathrm{mg}$ pertuzumab and $600 \mathrm{mg}$ trastuzumab in $15 \mathrm{~mL}$; maintenance dose: $600 \mathrm{mg}$ pertuzumab and trastuzumab in $10 \mathrm{~mL}$, and $2000 \mathrm{U} / \mathrm{mL}$ recombinant human hyaluronidase) provides a positive benefit-risk profile with comparable efficacy with $\mathrm{P}+\mathrm{H} \mathrm{IV}$ that is achieved with a manageable safety profile. In addition, PH FDC SC provides healthcare professionals with the possibility of administering treatment in the patient's home, as noted by the FDA [15]; an option that has become more important recently due to the COVID-19 pandemic. Home administration potentially reduces the risk of patients acquiring COVID-19 infection during hospital visits, a risk that could in turn lead to increased complications in patients with cancer [16]. The results further suggest that, in practice, no dose modifications are required according to covariates such as albumin, LBW, and race. 
a

SC

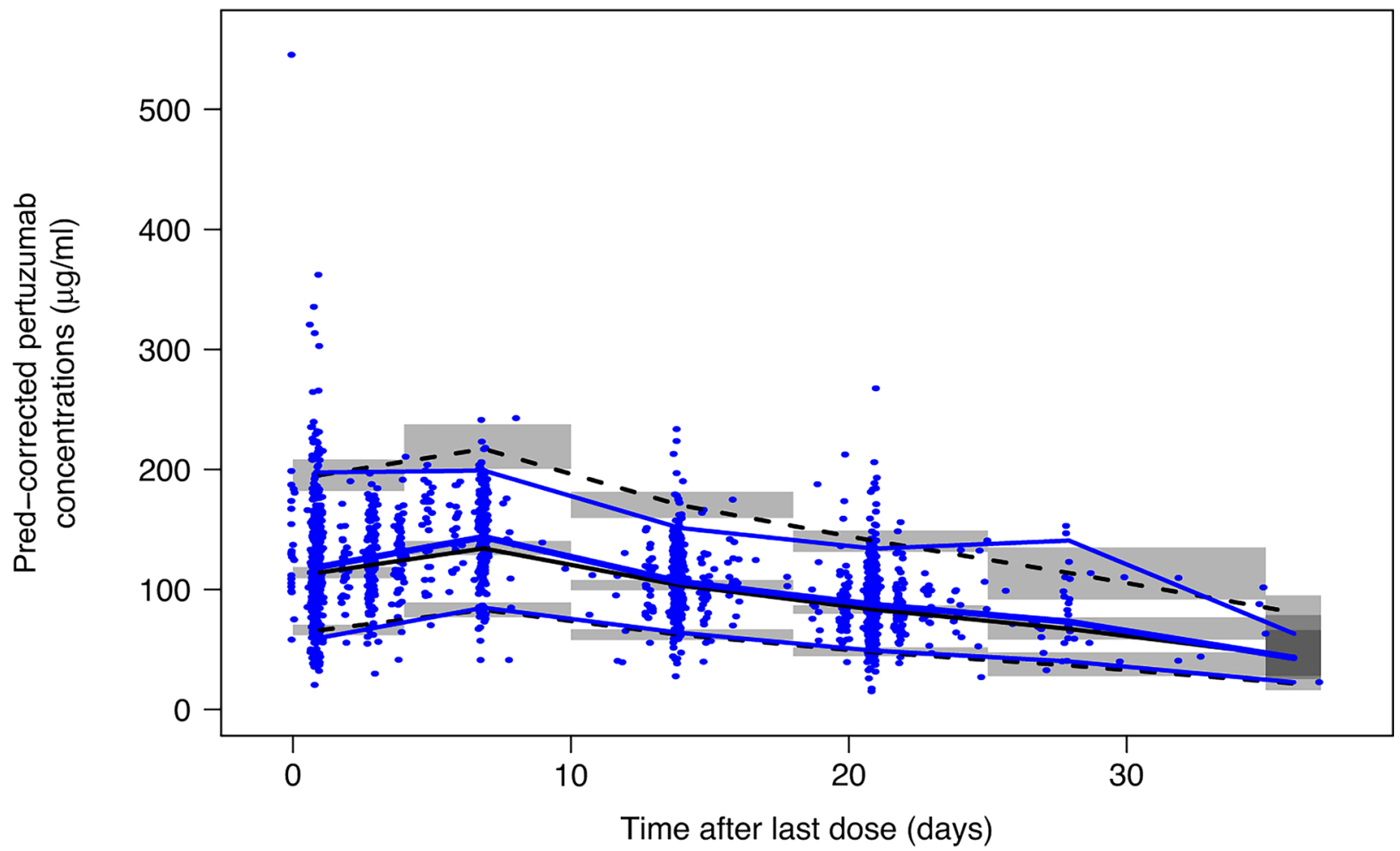

b

IV

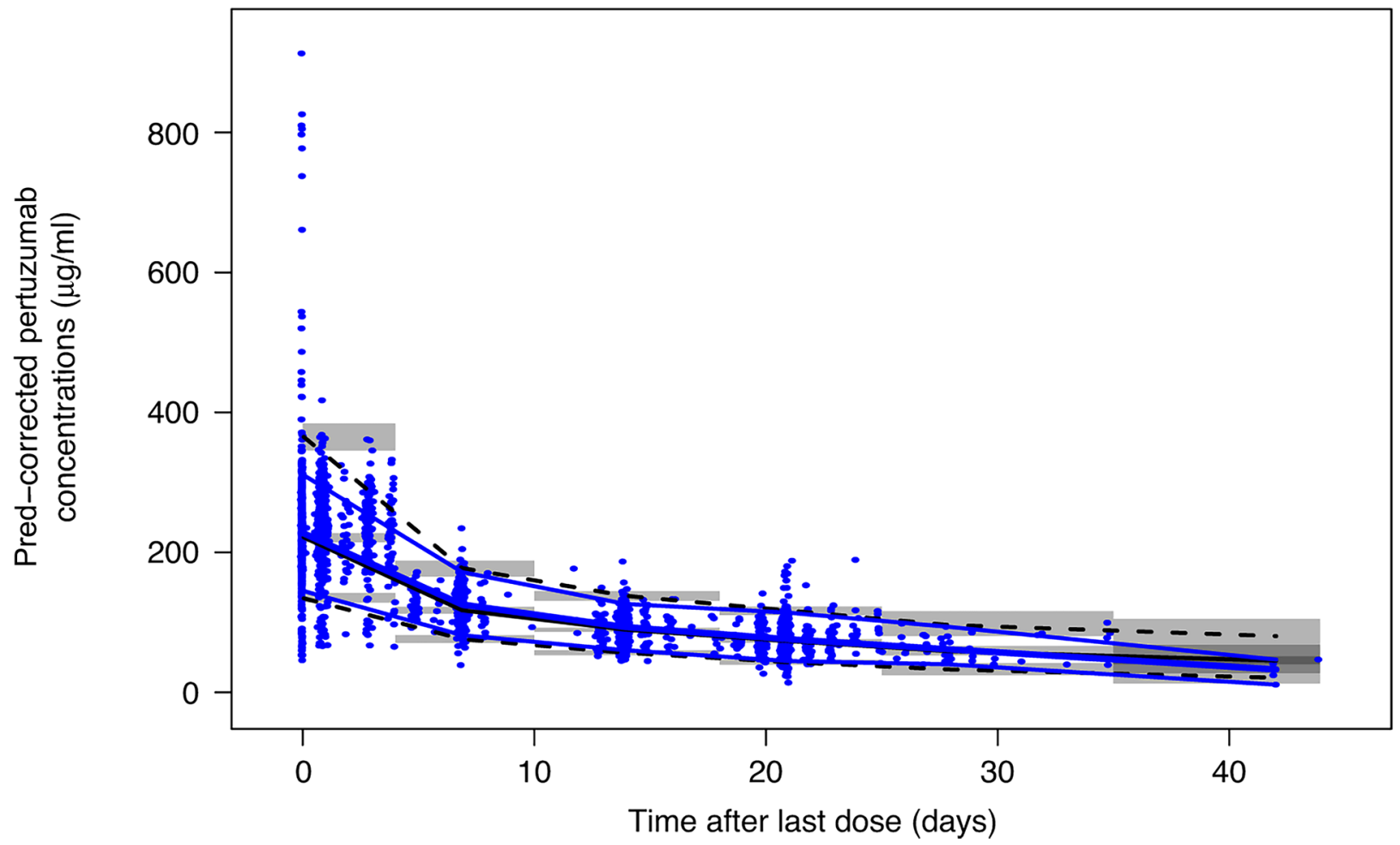


4Fig. 3 Prediction-corrected visual predictive check for pertuzumab after PH FDC SC (A) and P+H IV (B) administration. Blue dots represent observed pertuzumab concentrations. Blue lines represent the 5th, 50th, and 95th percentiles of observed pertuzumab concentrations. Black lines represent the 5th, 50th, and 95th percentiles of pertuzumab simulations. Gray bands represent the $95 \%$ prediction interval for corresponding black lines based on 500 simulations. $P+H I V$ is intravenous pertuzumab and trastuzumab. $P H F D C S C$ is fixed-dose combination of pertuzumab and trastuzumab for subcutaneous injection. Created using R version 3.6 .0 (2019-04-26) in RStudio version 1.2.1335

a

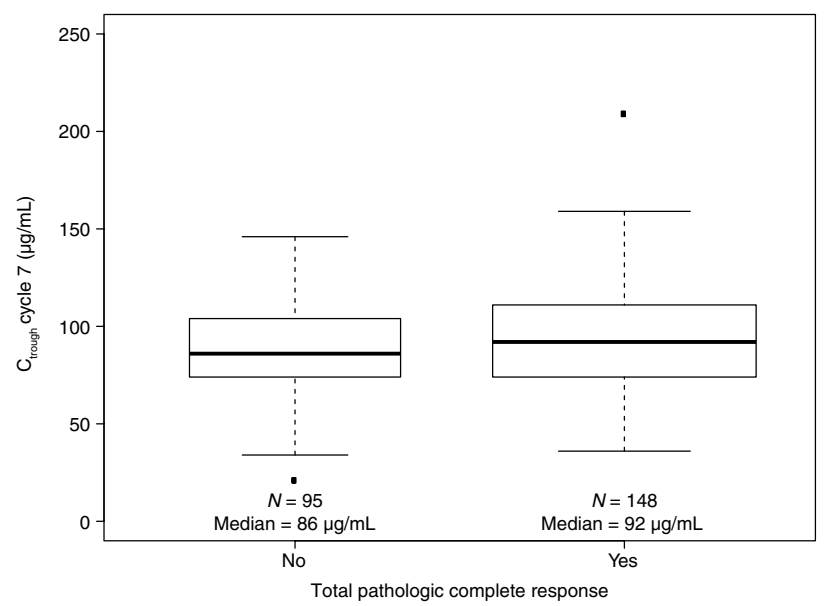

\section{Data-sharing statement}

Qualified researchers may request access to individual patient-level data through the clinical study data request platform: https://vivli.org/. Further details on Roche's criteria for eligible studies are available here: https://vivli.org/ members/ourmembers. For further details on Roche's Global Policy on the Sharing of Clinical Information and how to request access to related clinical study documents, see here:

b

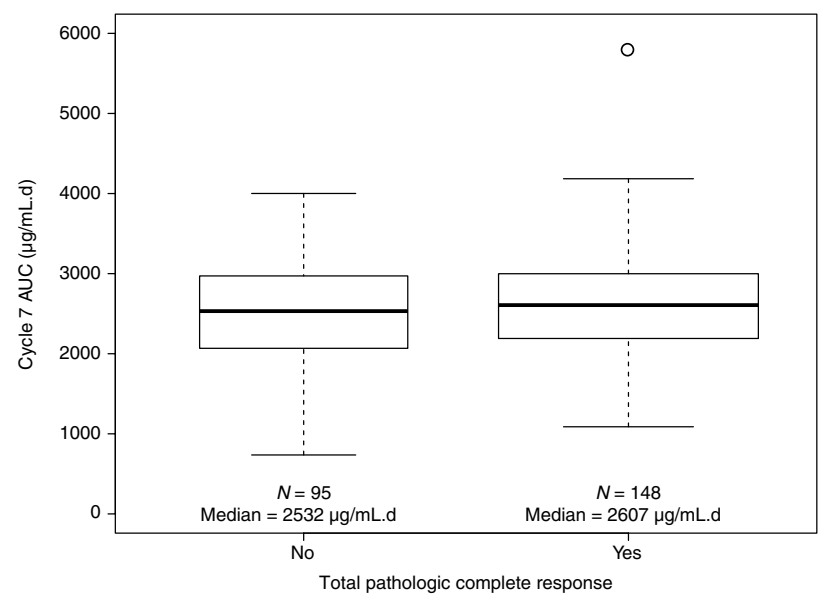

c

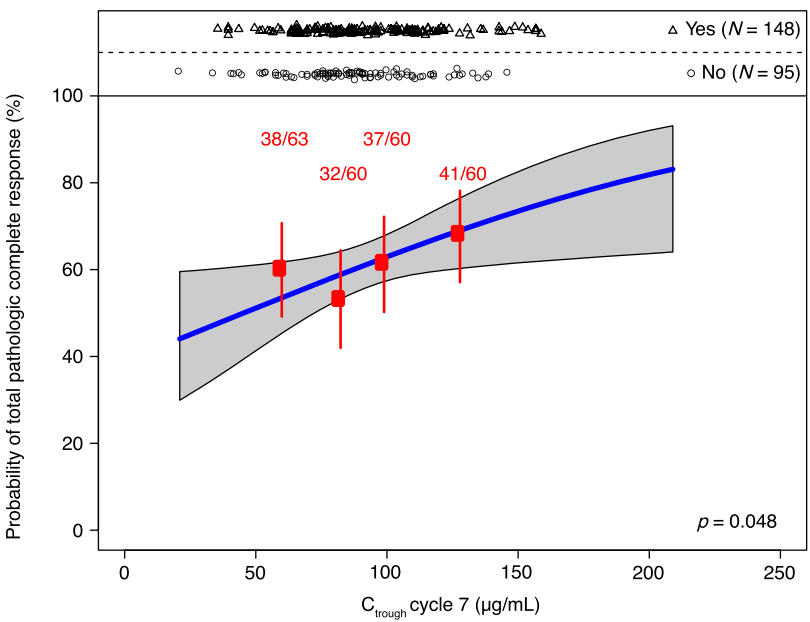

Fig. 4 Model-predicted cycle 7 pertuzumab $\mathrm{C}_{\text {trough }}$ (A) and AUC (B) by tpCR, and univariate exposure-response relationship (C). Boxplots show the median (solid bold line), the interquartile range (shaded boxes), 1.5 times the interquartile range (whiskers), and points outside the whiskers (solid points). Univariate analysis red points indicate the mean concentration and response probability per exposure quartile. Vertical error bars indicate $90 \%$ confidence bounds. Numbers above each bar indicates patients with the event and the total number of patients in the quartile group. The blue line rep- resents the fit of a logistic regression model. The shaded gray region indicates $90 \%$ confidence bands for the model fit. Points above the plot indicate model-predicted pertuzumab exposures in patients with a response versus exposures in patients with no response. $A U C$ is area under the curve for a dosing interval $(\operatorname{tau}=21)$. $C_{\max }$ is maximum concentration. $C_{\text {trough }}$ is trough concentration. $t p C R$ is total pathologic complete response. Created using R version 3.6.0 (2019-04-26) in RStudio version 1.2.1335 
Table 2 Total pathologic complete response rate and safety parameters stratified by pertuzumab cycle $7 \mathrm{C}_{\text {trough }}$ quartile in the PH FDC SC arm

\begin{tabular}{|c|c|c|c|c|c|}
\hline \multirow[b]{2}{*}{ Endpoint } & \multicolumn{5}{|c|}{ Pertuzumab cycle $7 \mathrm{C}_{\text {trough }}$ quartile, $\mu \mathrm{g} / \mathrm{mL}$} \\
\hline & $\begin{array}{l}\text { Q1 } \\
64[21-74] \\
(n=63)\end{array}$ & $\begin{array}{l}\text { Q2 } \\
82[75-89] \\
(n=60)\end{array}$ & $\begin{array}{l}\text { Q3 } \\
98[90-109] \\
(n=60)\end{array}$ & $\begin{array}{l}\text { Q4 } \\
122[110-209] \\
(n=60)\end{array}$ & $\begin{array}{l}\text { All } \\
89 \text { [21-209] } \\
(n=243)\end{array}$ \\
\hline tpCR & $38(60.3 \%)$ & $32(53.3 \%)$ & $37(61.7 \%)$ & $41(68.3 \%)$ & $148(60.9 \%)$ \\
\hline \multirow[t]{3}{*}{$\Delta$ tpCR } & $-0.7 \%(-14.2 \%, 12.9 \%)$ & $-7.6 \%(-21.5 \%, 6.2 \%)$ & $0.7 \%(-13.1 \%, 14.4 \%)$ & $7.4 \%(-6.3 \%, 21.0 \%)$ & - \\
\hline & \multicolumn{5}{|c|}{ Model-predicted pertuzumab Cycle $7 \mathrm{C}_{\max }$ quartile, $\mu \mathrm{g} / \mathrm{mL}$} \\
\hline & $\begin{array}{l}\text { Q1 } \\
{[51-127]} \\
(n=61)\end{array}$ & $\begin{array}{l}\text { Q2 } \\
{[128-150]} \\
(n=61)\end{array}$ & $\begin{array}{l}\text { Q3 } \\
{[151-178]} \\
(n=62)\end{array}$ & $\begin{array}{l}\text { Q4 } \\
{[180-336]} \\
(n=59)\end{array}$ & $\begin{array}{l}\text { All } \\
{[51-336]} \\
(n=243)\end{array}$ \\
\hline Grade $\geq 3 \mathrm{AE}$ & $13(21.3 \%)$ & $24(39.3 \%)$ & $14(22.6 \%)$ & $19(32.2 \%)$ & $70(28.8 \%)$ \\
\hline Serious AE & $7(11.5 \%)$ & $9(14.8 \%)$ & $5(8.1 \%)$ & $4(6.8 \%)$ & $25(10.3 \%)$ \\
\hline Grade $\geq 3$ diarrhea & $2(3.3 \%)$ & $5(8.2 \%)$ & $3(4.8 \%)$ & $3(5.1 \%)$ & $13(5.3 \%)$ \\
\hline Grade $\geq 3$ neutropenia & $5(8.2 \%)$ & $13(21.3 \%)$ & $5(8.1 \%)$ & $10(16.9 \%)$ & $33(13.6 \%)$ \\
\hline Cardiac toxicity & $7(11.5 \%)$ & $5(8.2 \%)$ & $7(11.3 \%)$ & $3(5.1 \%)$ & $22(9.1 \%)$ \\
\hline $\begin{array}{l}\text { IRR (any grade) within } 24 \mathrm{~h} \text {, } \\
\text { related to PH FDC SC }\end{array}$ & $5(8.2 \%)$ & $6(9.8 \%)$ & $10(16.1 \%)$ & $3(5.1 \%)$ & $24(9.9 \%)$ \\
\hline $\begin{array}{l}\text { Hypersensitivity/anaphylaxis (any } \\
\text { grade), related to PH FDC SC }\end{array}$ & $2(3.3 \%)$ & 0 & 0 & 0 & $2(0.8 \%)$ \\
\hline
\end{tabular}

Numbers in square brackets indicate the range of pertuzumab cycle $7 \mathrm{C}_{\text {trough/max }}$

$A E$ is adverse event, $C_{\max }$ is maximum concentration, $C_{\text {trough }}$ is trough concentration, $I R R$ is injection-related reaction, $P+H I V$ is intravenous pertuzumab and trastuzumab, $P H F D C S C$ is fixed-dose combination of pertuzumab and trastuzumab for subcutaneous injection, $t p C R$ is total pathologic complete response, $\triangle t p C R$ is difference from overall rate in IV arm (for comparison, in the P+H IV arm, rate of tpCR was 150/246 or $61.0 \%)$

https://www.roche.com/research_and_development/who_ we_are_how_we_work/clinical_trials/our_commitment_ to_data_sharing.htm.

Supplementary Information The online version contains supplementary material available at https://doi.org/10.1007/s00280-021-04296-0.

Acknowledgments We would like to thank all the patients who participated in the trial, and their families, the investigators, clinicians, and research staff at the 106 centers in 19 countries. We would also like to thank Professor Giuseppe Viale, MD, of the Department of Pathology, European Institute of Oncology, University of Milan, Milan, Italy for screening candidate patients for the study (central pathology confirmation of eligibility criteria), and for reviewing the pathology reports for the tpCR endpoint. Support for third-party writing assistance for this manuscript, furnished by Daniel Clyde, $\mathrm{PhD}$, of Health Interactions, was provided by F. Hoffmann-La Roche Ltd.

Funding This study is sponsored by F. Hoffmann-La Roche Ltd and Genentech, Inc.

\section{Declarations}

Conflict of interest Bei Wang, Matts Kågedal, Chunze Li, and Whitney P. Kirschbrown are employees of Genentech, Inc. and own stock in Roche Holding Ltd. Angelica L. Quartino was an employee of Genentech, Inc. at the time of the study. Sandhya Girish was an employee of Genentech, Inc. and owned stock in Roche Holding Ltd at the time of the study. Rong Deng is an independent consultant for
Genentech, Inc. Tanja Badovinac Crnjevic and Monika Kaewphluk are employees of and hold stocks in Roche Holding Ltd. Stefanie Hennig is an employee of Cetera, Inc., and has received consulting fees from Genentech, Inc. in connection with this work. Tanja Badovinac Crnjevic and Whitney P. Kirschbrown are patent holders for the fixeddose combination of pertuzumab and trastuzumab for subcutaneous injection. All authors received research support in the form of thirdparty writing assistance for this manuscript from Health Interactions, provided by F. Hoffmann-La Roche Ltd.

Research involving human participants FeDeriCa was conducted in conformance with Good Clinical Practice guidelines and the Declaration of Helsinki. The protocol was reviewed and approved by the institutional review board or ethics committee at each study site.

Informed consent All patients provided written informed consent.

Open Access This article is licensed under a Creative Commons Attribution 4.0 International License, which permits use, sharing, adaptation, distribution and reproduction in any medium or format, as long as you give appropriate credit to the original author(s) and the source, provide a link to the Creative Commons licence, and indicate if changes were made. The images or other third party material in this article are included in the article's Creative Commons licence, unless indicated otherwise in a credit line to the material. If material is not included in the article's Creative Commons licence and your intended use is not permitted by statutory regulation or exceeds the permitted use, you will need to obtain permission directly from the copyright holder. To view a copy of this licence, visit http://creativecommons.org/licenses/by/4.0/. 


\section{References}

1. Nahta R, Hung MC, Esteva FJ (2004) The HER-2-targeting antibodies trastuzumab and pertuzumab synergistically inhibit the survival of breast cancer cells. Cancer Res 64(7):2343-2346

2. Scheuer W, Friess T, Burtscher H, Bossenmaier B, Endl J, Hasmann M (2009) Strongly enhanced antitumor activity of trastuzumab and pertuzumab combination treatment on HER2-positive human xenograft tumor models. Cancer Res 69(24):9330-9336

3. Junttila TT, Akita RW, Parsons K et al (2009) Ligand-independent HER2/HER3/PI3K complex is disrupted by trastuzumab and is effectively inhibited by the PI3K inhibitor GDC-0941. Cancer Cell 15(5):429-440

4. Adams CW, Allison DE, Flagella K et al (2006) Humanization of a recombinant monoclonal antibody to produce a therapeutic HER dimerization inhibitor, pertuzumab. Cancer Immunol Immunother 55(6):717-727

5. Diermeier-Daucher S, Hasmann M, Brockhoff G (2008) Flow cytometric FRET analysis of erbB receptor interaction on a cellby-cell basis. Ann N Y Acad Sci 1130:280-286

6. Gianni L, Pienkowski T, Im YH et al (2012) Efficacy and safety of neoadjuvant pertuzumab and trastuzumab in women with locally advanced, inflammatory, or early HER2-positive breast cancer (NeoSphere): a randomised multicentre, open-label, phase 2 trial. Lancet Oncol 13(1):25-32

7. Gianni L, Pienkowski T, Im YH et al (2016) 5-year analysis of neoadjuvant pertuzumab and trastuzumab in patients with locally advanced, inflammatory, or early-stage HER2-positive breast cancer (NeoSphere): a multicentre, open-label, phase 2 randomised trial. Lancet Oncol 17(6):791-800

8. von Minckwitz G, Procter M, de Azambuja E et al (2017) Adjuvant pertuzumab and trastuzumab in early HER2-positive breast cancer. N Engl J Med 377(2):122-131

9. Piccart M, Procter M, Fumagalli D et al (2019) Interim overall survival analysis of APHINITY (BIG 4-11): a randomized multicenter, double-blind, placebo-controlled trial comparing chemotherapy plus trastuzumab plus pertuzumab versus chemotherapy plus trastuzumab plus placebo as adjuvant therapy in patients with operable HER2-positive early breast cancer. Cancer Res 80(4 Suppl):GS1-04

10. Baselga J, Cortés J, Kim SB et al (2012) Pertuzumab plus trastuzumab plus docetaxel for metastatic breast cancer. N Engl J Med 366(2):109-119

11. Swain SM, Kim S-B, Cortés J et al (2013) Pertuzumab, trastuzumab, and docetaxel for HER2-positive metastatic breast cancer (CLEOPATRA study): overall survival results from a randomised, double-blind, placebo-controlled, phase 3 study. Lancet Oncol 14(6):461-471

12. Swain SM, Baselga J, Kim SB et al (2015) Pertuzumab, trastuzumab, and docetaxel in HER2-positive metastatic breast cancer. N Engl J Med 372(8):724-734

13. Swain SM, Miles D, Kim SB et al (2020) Pertuzumab, trastuzumab, and docetaxel for HER2-positive metastatic breast cancer (CLEOPATRA): end-of-study results from a double-blind, randomised, placebo-controlled, phase 3 study. Lancet Oncol 21:519-530

14. O'Shaughnessy J, Sousa S, Cruz J et al (2020) Patient (pt) preference for the pertuzumab-trastuzumab fixed-dose combination for subcutaneous use (PH FDC SC) in HER2-positive early breast cancer (EBC): primary analysis of the open-label, randomised crossover PHranceSCa study. Ann Oncol 31(4 Suppl):165MO

15. Food and Drug Administration (FDA) FDA approves breast cancer treatment that can be administered at home by health care professional [press release]. 29 June 2020. https://www.fda.gov/newsevents/press-announcements/fda-approves-breast-cancer-treat ment-can-be-administered-home-health-care-professional. Accessed Sept 2020

16. Wardley A, Canon JL, Elsten L et al (2021) Flexible care in breast cancer. ESMO Open 6(1):100007

17. Ismael G, Hegg R, Muehlbauer $S$ et al (2012) Subcutaneous versus intravenous administration of (neo)adjuvant trastuzumab in patients with HER2-positive, clinical stage I-III breast cancer (HannaH study): a phase 3, open-label, multicentre randomised trial. Lancet Oncol 13(9):869-878

18. Jackisch C, Kim SB, Semiglazov V et al (2015) Subcutaneous versus intravenous formulation of trastuzumab for HER2-positive early breast cancer: updated results from the phase III HannaH study. Ann Oncol 26(2):320-325

19. Jackisch C, Hegg R, Stroyakovskiy D et al (2016) HannaH phase III randomised study: association of total pathological complete response with event-free survival in HER2-positive early breast cancer treated with neoadjuvant-adjuvant trastuzumab after 2 years of treatment-free follow-up. Eur J Cancer 62:62-75

20. Jackisch C, Stroyakovskiy D, Pivot X et al (2019) Subcutaneous vs intravenous trastuzumab for patients with ERBB2-positive early breast cancer: final analysis of the HannaH phase 3 randomized clinical trial. JAMA Oncol 5(5):e190339

21. Pivot X, Gligorov J, Müller V et al (2013) Preference for subcutaneous or intravenous administration of trastuzumab in patients with HER2-positive early breast cancer (PrefHer): an open-label randomised study. Lancet Oncol 14(10):962-970

22. Pivot X, Gligorov J, Müller V et al (2014) Patients' preferences for subcutaneous trastuzumab versus conventional intravenous infusion for the adjuvant treatment of HER2-positive early breast cancer: final analysis of 488 patients in the international, randomized, two-cohort PrefHer study. Ann Oncol 25(10):1979-1987

23. Pivot X, Verma S, Fallowfield L et al (2017) Efficacy and safety of subcutaneous trastuzumab and intravenous trastuzumab as part of adjuvant therapy for HER2-positive early breast cancer: final analysis of the randomised, two-cohort PrefHer study. Eur J Cancer 86:82-90

24. Gligorov J, Ataseven B, Verrill M et al (2017) Safety and tolerability of subcutaneous trastuzumab for the adjuvant treatment of human epidermal growth factor receptor 2-positive early breast cancer: SafeHer phase III study's primary analysis of 2573 patients. Eur J Cancer 82:237-246

25. Gligorov J, Curigliano G, Muller V et al (2017) Switching between intravenous and subcutaneous trastuzumab: safety results from the PrefHer trial. Breast 34:89-95

26. Jung KH, Ataseven B, Verrill M et al (2018) Adjuvant subcutaneous trastuzumab for HER2-positive early breast cancer: subgroup analyses of safety and active medical conditions by body weight in the SafeHer phase III study. Oncologist 23(10):1137-1143

27. Garg A, Quartino A, Li J et al (2014) Population pharmacokinetic and covariate analysis of pertuzumab, a HER2-targeted monoclonal antibody, and evaluation of a fixed, non-weight-based dose in patients with a variety of solid tumors. Cancer Chemother Pharmacol 74(4):819-829

28. Kirschbrown WP, Wynne C, Kågedal M et al (2019) Development of a subcutaneous fixed-dose combination of pertuzumab and trastuzumab: results from the phase Ib dose-finding study. J Clin Pharmacol 59(5):702-716

29. Quartino AL, Hillenbach C, Li J et al (2016) Population pharmacokinetic and exposure-response analysis for trastuzumab administered using a subcutaneous "manual syringe" injection or intravenously in women with HER2-positive early breast cancer. Cancer Chemother Pharmacol 77(1):77-88

30. Tan AR, Im S-A, Mattar A et al (2021) Fixed-dose combination of pertuzumab and trastuzumab for subcutaneous injection plus chemotherapy in HER2-positive early breast cancer (FeDeriCa): 
a randomised, open-label, multicentre, non-inferiority, phase 3 study. Lancet Oncol 22(1):85-97

31. Quartino AL, Li H, Jin JY et al (2017) Pharmacokinetic and exposure-response analyses of pertuzumab in combination with trastuzumab and docetaxel during neoadjuvant treatment of HER2 early breast cancer. Cancer Chemother Pharmacol 79(2):353-361

32. Kirschbrown WP, Kågedal M, Wang B et al (2019) Pharmacokinetic and exploratory exposure-response analysis of pertuzumab in patients with operable HER2-positive early breast cancer in the APHINITY study. Cancer Chemother Pharmacol 83(6):1147-1158
33. Keizer RJ, Huitema AD, Schellens JH, Beijnen JH (2010) Clinical pharmacokinetics of therapeutic monoclonal antibodies. Clin Pharmacokinet 49(8):493-507

34. Baverel P, Roskos L, Tatipalli M et al (2019) Exposure-response analysis of overall survival for tremelimumab in unresectable malignant mesothelioma: the confounding effect of disease status. Clin Transl Sci 12(5):450-458

Publisher's Note Springer Nature remains neutral with regard to jurisdictional claims in published maps and institutional affiliations. 